\title{
Defect Formation on Surfaces Bombarded by Energetic Multiply Charged Proteins: Implications for the Conformation of Gas-Phase Electrosprayed Ions
}

\author{
P. A. Sullivan, J. Axelsson,* S. Altmann, A. P. Quist, B. U. R. Sunqvist, \\ and C. T. Reimann \\ Division of Ion Physics, Department of Radiation Sciences, Uppsala University, Uppsala, Sweden
}

\begin{abstract}
Indirect information on the conformation of highly charged molecular ions may be obtained by monitoring their collisional cross sections and the course of simple gas-phase reactions such as hydrogen-deuterium exchange. In this work, another indirect but more visually oriented approach is explored: electrosprayed protein ions are accelerated toward a highly oriented pyrolytic graphite surface and the resulting single-ion defects are imaged by scanning force and tunneling microscopy. All protein impacts generated shallow hillocks: the shapes depended on the identity and charge state of the incident protein. Lysozyme and myoglobin, both compact, globular proteins in the native state, produced compact, almost circular hillocks. However, hillocks generated by myoglobin that had been denatured in the solution phase were elongated, and the elongation was positively correlated with the charge state of the ion. It appears that structural information about gas-phase multiply charged proteins can be derived from imprints generated by energetic protein impacts on surfaces. ( ( Am Soc Mass Spectrom 1996, 7, 329-341)
\end{abstract}

$\mathrm{E}$ lectrospray ionization (ESI) techniques for applications in mass spectrometry of large bioorganic molecules have flourished in the last few years. ESI is a "soft" technique that works well for fragile and thermally labile bioorganic molecules. Electrosprayed molecular ions commonly are created in high charge states, which means that a simple mass spectrometer with a limited range of mass-to-charge ratio may be employed for the mass analysis. Finally, molecules with an extremely broad range of mass may be ionized. Several reviews have appeared on the capabilities and prospects of ESI and on the ionization mechanism [1-3]. A representative list of proteins that have been ionized by electrospray has been given in the mass range 409-133,000 u [4], but even more massive molecules have been ionized. For example, coliphage T4 DNA of mass exceeding $10^{8} \mathrm{u}$ has been electrosprayed successfully and detected in charge states of hundreds of thousands of unit charges [5].

Strong correlations have been observed between solution-phase molecular conformation and resulting

Address reprint requests to Dr. P. A. Sullivan, Uppsala University, Division of Ion Physics, Department of Radiation Sciences, Box 535, 75121 Uppsala, Sweden.

* Current address: Department of Chemistry, University of Warwick, Coventry CV4 7AL, United Kingdom.
ESI charge-state distributions. The maximum number of protons adducted to electrosprayed proteins often is correlated with the number of basic amino-acid residues in the molecule [4]. However, for proteins stabilized by disulfide linkages between cysteine bases, the maximum charge state observed frequently falls short of what is predicted by this principle [6]. The opening of these disulfide linkages, accompanied by the unfolding of the protein in solution, results in a drastic increase in the maximum charge state [6]. It is suspected that the unfolding process "exposes" more basic amino-acid residues and allows a higher charge state to be achieved.

Disulfide linkages are not the only source of structural stability in proteins. Changes in $\mathrm{pH}$ [7], solvent [8], and temperature [9], which can alter the configuration of hydrogen bonding in the protein and also alter the nature and effectiveness of the polar and nonpolar interactions to stabilize the protein, also can result in solution-phase denaturation. Again, such treatments are connected with degrees of change in the resulting ESI charge-state distributions. This correlation is often assumed to work in reverse: changes in ESI charge-state distributions imply a change in the protein conformation in the solution phase [10].

In spite of the compelling correlations that have been observed between solution-phase structure and 
ESI charge-state distributions, it is not known in a rigorous fashion whether the solution-phase structure corresponds to the gas-phase structure. During electrospraying, it is possible that the solution-phase environment changes and that this affects both the solutionphase and eventual gas-phase structures. Also, after the gas phase is reached, Coulomb forces between the adducted charges may become much more important and may serve to additionally modify the gas-phase structure [11]. These complicated considerations have stimulated new research aimed at analysis of the behavior of electrosprayed ions after they have reached the gas phase.

In one class of such studies, the reactivity of gasphase highly charged proteins with other species was monitored [12, 13]. Proton transfer (charge-state reduction) rates of disulfide-linked proteins in the gas phase depended on whether or not the disulfide links had been intact in the solution phase [14, 15]. In contrast, for proteins not stabilized by disulfide bonds, proton transfer rates of the highly charged ions appeared similar for species that display different ESI chargestate distributions [14], which suggests that this class of proteins may end up displaying similar gas-phase conformations despite their apparently different solution-phase conformations. The hydrogen-deuterium exchange behavior of highly charged protein ions stored in a Fourier-transform ion cyclotron resonance trap also has been studied to provide clues to gas-phase structure $[16,17]$. For cytochrome $c$, some charge states displayed multiple conformations and, furthermore, the highest charge state did not appear to possess the most open structure [17]. This result suggests that charge state is not always simply correlated with gasphase structure. By contrast, in still other studies of gas-phase interactions, the collision cross sections for several species of highly charged proteins were found to increase with the charge state of the protein, both for disulfide-stabilized [18, 19] and non-disulfidestabilized proteins [18-20].

It is difficult to draw any rigorous conclusions from the ensemble of results obtained in these gas-phase studies. Consequently, there exists a need for a complimentary approach to determining the gas-phase conformation of proteins. In the work described herein we have attempted to provide new information about the conformational state of electrosprayed molecular ions by accelerating them to kinetic energies of about 200 $\mathrm{keV}$ and impacting them onto a highly oriented pyrolytic graphite (HOPG) surface. The resulting defects - each one created by an individual incident molecular ion-are innaged by means of scanning force microscopy (SFM) and scanning tunneling microscopy (STM). We assumed that compact projectiles would produce compact surface features, whereas more extended or "sticklike" projectiles would generate oblong hillocks with a distribution of lengths because of their random orientations upon impact with the target surface.

\section{Experimental}

Energetic protein ions were generated by using the multiply charged macromolecular accelerator (MUMMA) at Uppsala. This apparatus has been described in detail elsewhere ([21-28] and Håkansson, P.; Della-Negra, S.; Mouffron, J. P.; Waast, B.; Sullivan, P. A., unpublished). MUMMA is adapted from a commercial mass spectrometer (Perceptive/Vestec Products, Houston, TX) which consists of an ESI source [29] and a quadrupole mass spectrometer (QMS).

All proteins employed in this study were obtained commercially (Sigma Chemical Co., St. Louis, MO) and were used without further purification. Horse heart myoglobin (MYO) solutions were prepared to be electrosprayed by dissolving $\sim 10^{-5}-\mathrm{M}$ concentrations of MYO in pure deionized $\mathrm{H}_{2} \mathrm{O}$. Apomyoglobin (AMYO) and lysozyme (LYS) solutions were prepared by combining $48 \%$ of $\sim 10^{-5}-\mathrm{M}$ concentrations of the native protein with $48 \%$ methanol and $4 \%$ acetic acid. Protein ESI mass spectra obtained with our instrument under typical conditions (solution flow rate $=1 \mu \mathrm{L} \mathrm{min}{ }^{-1}$; repeller voltage $\approx 10 \mathrm{~V}$ ) are displayed in Figure 1 . To achieve high particle count rates at the target, in the range of $10^{3}-10^{5}$ particles per second, the resolution of the QMS was degraded, but charge-state peaks were always well resolved. Irradiations generally took a few hours to produce about 10 impacts per micrometer squared within a beam spot area of about $2 \mathrm{~mm}^{2}$. Parameters of the projectiles employed in this study are displayed in Table 1.

Once the gas-phase protein ions were created and charge-state selected, they were accelerated onto freshly cleaved HOPG (Advanced Ceramics Corp., Cleveland, $\mathrm{OH}$ ). In some cases, experiments also were performed on freshly cleaved mica (AshevilleSchoonmaker Mica Co., Newport News, VA). The number distribution of secondary electrons emitted from the target during an ensemble of protein ion impacts is a function of the mass and velocity of the incident ions [24]. These secondary electron distributions were monitored during experiments to ensure

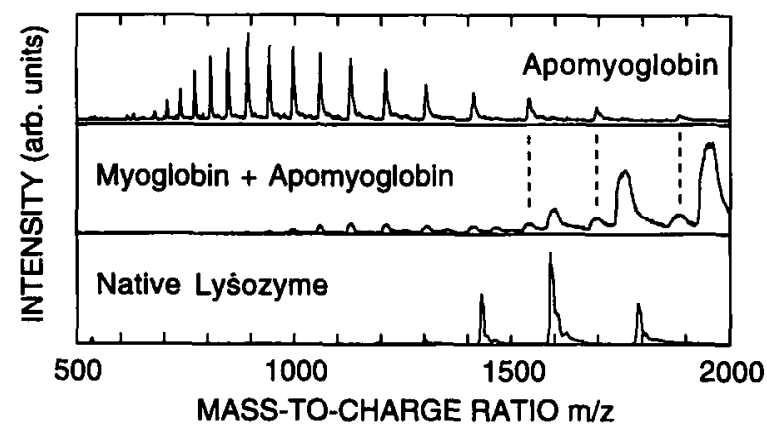

Figure 1. Electrospray ionization mass spectra of proteins used in surface impact studies. The dashed lines in the middle spectrum show three of the apomyoglobin charge-state species that coexist with the myoglobin species, which show the mass difference between the two proteins apomyoglobin and myoglobin. 
Table 1. Parameters of the protein projectiles used in this study ${ }^{\circ}$

\begin{tabular}{|c|c|c|c|c|}
\hline $\begin{array}{l}\text { Incident protein } \\
\text { (abbreviation) }\end{array}$ & $\begin{array}{c}\text { Mass }(\mathrm{M}+n \mathrm{H}) \\
(\mathrm{u})\end{array}$ & Charge & $\begin{array}{c}\text { Energy } \\
\text { (keV) }\end{array}$ & $\begin{array}{l}\text { Velocity } \\
(\mathrm{km} / \mathrm{s})\end{array}$ \\
\hline Apomyoglobin (AMYO ${ }^{18+}$ ) & $16951+18$ & +18 & $\overline{7176}$ & 44.8 \\
\hline Apomyoglobin (AMYO ${ }^{16+}$ ) & $16951+16$ & +16 & 176 & 44.8 \\
\hline Apomyoglobin (AMYO $\left.{ }^{14+}\right)^{*}$ & $16951+14$ & +14 & 176 & 44.8 \\
\hline Apomyoglobin (AMYO ${ }^{14+}$ ) & $16951+14$ & +14 & 252 & 53.6 \\
\hline Apomyoglobin $\left(\mathrm{AMYO}^{9+}\right.$ ) & $16951+9$ & +9 & 176 & 44.8 \\
\hline Myoglobin (MYO ${ }^{10+}$ ) & $17567+10$ & +10 & 198 & 46.6 \\
\hline Lysozyme $\left(\text { LYS }^{9+}\right)^{\star}$ & $14306+9$ & +9 & 146 & 44.4 \\
\hline
\end{tabular}

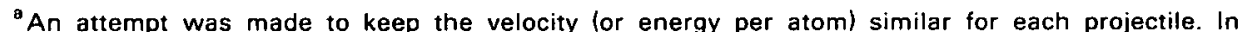
addition, some experiments were repeated and the apomyoglobin charge state $Q=+14$ was run at two different energies: 176 and $252 \mathrm{keV}$. The target was HOPG. The asterisk (*) indicates that the experiment also was performed by using a mica target.
}

that proteins that impacted the target were intact $[23$, 24].

After irradiation, the samples were analyzed with ambient SFM on a NanoScope III (Digital Instruments, Santa Barbara, CA). In TappingMode ${ }^{\mathrm{TM}}$ SFM (TM-SFM), the surfaces were probed with NanoProbe ${ }^{\mathrm{TM}}$ silicon tips with a nominal radius of curvature of $10 \mathrm{~nm}$ and cone angle of $36^{\circ}$. The TM-SFM is designed to drastically reduce the large normal and shear contact forces [30] that typically act between the probe tip and the surface in the conventional continuous repulsive contact mode SFM (CM-SFM) [31]. In addition, in selected cases a surface was imaged a second time by using a different tip or a different microscope mode, which included CM-SFM or STM. For STM, mechanically formed PtIr tunneling tips were employed with an applied tunneling bias potential of $20 \mathrm{mV}$.

\section{Results}

Figure 2 shows typical $500 \times 500$-nm TM-SFM and STM images of surface hillocks generated by energetic AMYO projectiles incident on HOPG. The hillocks are quite distinctive and are not observed on unbombarded HOPG. Although evidence for a small crater in the top of each hillock has been seen in STM images of hillocks generated by $C_{60}$ (fullerene) impacts on HOPG ([32] and Bräuchle, G.; Richard-Schneider, S.; Illig, D.; Bech, R. D.; Schreiber, H.; Kappes, M. M., unpublished), the features generated by protein impacts on HOPG appear as simple hillocks for all imaging techniques employed, which include TM-SFM, CM-SFM, and STM. Shown below each image are corresponding height, basal width, and basal length histograms of the hillocks taken from a series of images that include the ones shown here. The hillocks imaged with TM-SFM are on the order of $1 \mathrm{~nm}$ in height, $20 \mathrm{~nm}$ in width, and 20-60 $\mathrm{nm}$ in length. The hillocks imaged with STM were distributed differently in height and appeared to be narrower and shorter than hillocks imaged with TM-SFM.
We also probed irradiated mica samples for defect formation. Figure 3 compares typical defects induced in mica and HOPG by energetic AMYO projectiles. Hillocks observed on the mica samples displayed similar statistical length and width distributions (data not shown) as compared to hillocks observed on the HOPG samples. In contrast to the hillocks observed on HOPG, the hillocks on mica could be described as "coffee bean" shaped because they displayed craters in the center oriented along the long axis of the hillock (Figure 3). Hillock heights were distributed around slightly lower values for mica (data not shown) than for HOPG, consistent with the loss of more material from mica due to crater formation.

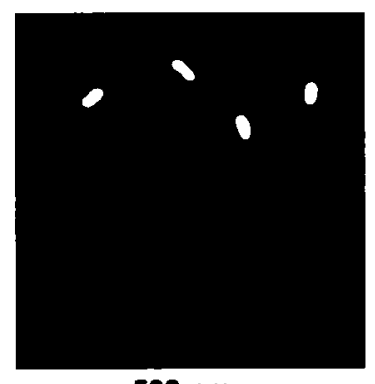

$500 \mathrm{~nm}$
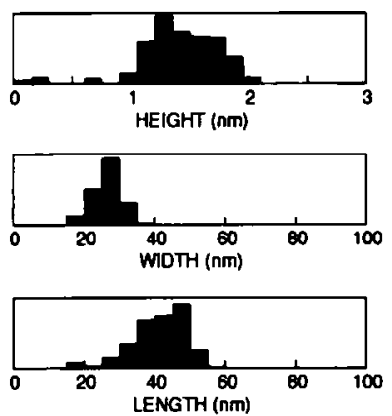

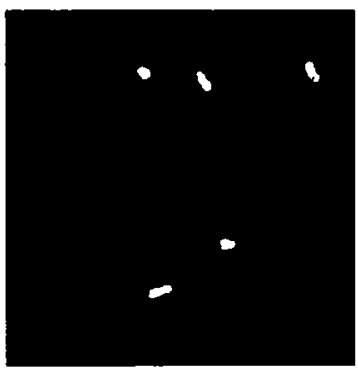

$500 \mathrm{~nm}$
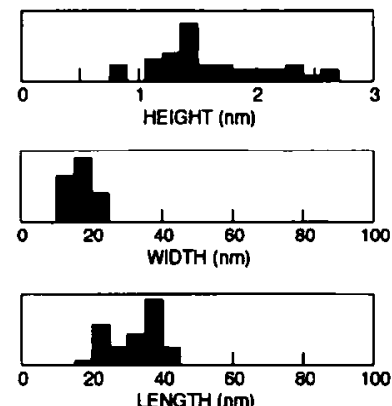

Figure 2. Comparison of TM-SFM (left) and STM (right) images of the same HOPG sample, which had been bombarded by 252-keV apomyoglobin in charge state $Q=+14$. Histograms of hillock dimensions show that lateral dimensions of the hillocks appear significantly wider in TM-SFM than in STM. (TM-SFM: 232 hillocks analyzed; STM: 40 hillocks analyzed.) 


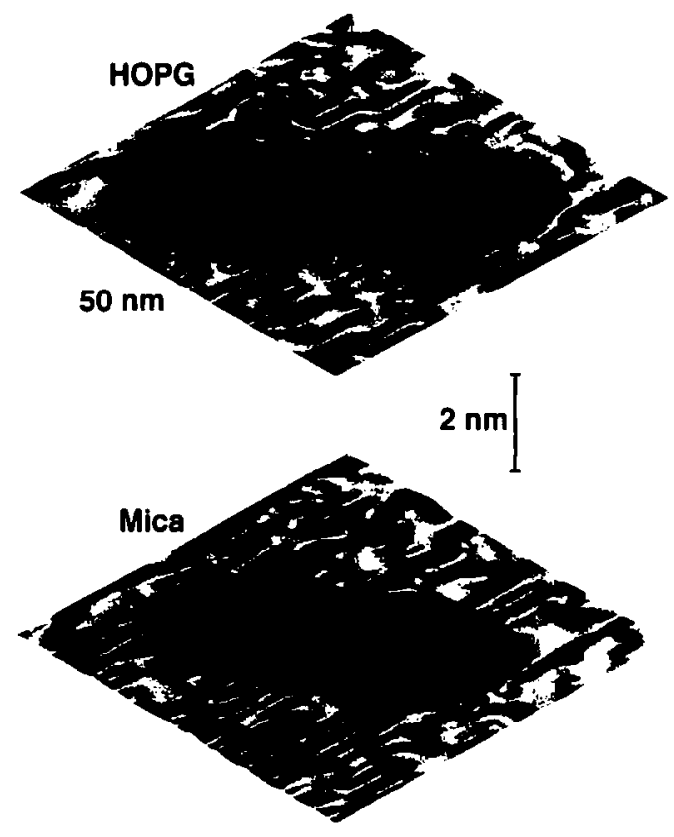

Figure 3. "Coffee beans" on mica. TM-SFM images of HOPG and mica bombarded by apomyoglobin $(Q=+14)$ at $176 \mathrm{keV}$. The length and width dimensions of the features were very similar. However, the mica bumps showed evidence of cratering, whereas the HOPG bumps did not.
The heights of the hillocks on HOPG depended on the kinetic energy of the impacting particle. For example, comparing the histograms shown in Figures 2 and 4 , it is seen that a projectile with a kinetic energy of $252 \mathrm{keV}$ (Figure 2) produced taller hillocks than the same projectile with a kinetic energy of $176 \mathrm{keV}$ (Figure 4).

The lateral and height dimensions of the hillocks depended on the parameters of the incident protein ions. For instance, the shapes of the hillocks from the impacts of AMYO depended on the charge state of the ion (Figure 4). AMYO ${ }^{18+, 16+, 14+}$ projectiles produced hillocks with oblong, "ricelike" shapes (Figure 4). In contrast, the projectiles of the lower charge states, which include $\mathrm{AMYO}^{9+}, \mathrm{MYO}^{10+}$, and $\mathrm{LYS}^{9+}$, generated hillocks that were still slightly oblong, but which were closer to being circular (Figures 4 and 5). Moreover, $\mathrm{AMYO}^{y+}, \mathrm{MYO}^{10+}$, and $\mathrm{LYS}^{y+}$ generated slightly wider and distinctly taller hillocks relative to $\mathrm{AMYO}^{18+, 16+, 14+}$. $\mathrm{LYS}^{9+}$ generated the roundest, tallest hillocks, followed by $\mathrm{MYO}^{10+}$ and $\mathrm{AMYO}^{9+}$, which generated successively lower and slightly more oblong hillocks.

The width distributions of all the hillocks were typically narrow and symmetric. The length and height distributions from the samples bombarded with $\mathrm{AMYO}^{9+}, \mathrm{MYO}^{10+}$, and $\mathrm{LYS}^{9+}$ were also nearly symmetric. In contrast, the length and height distributions
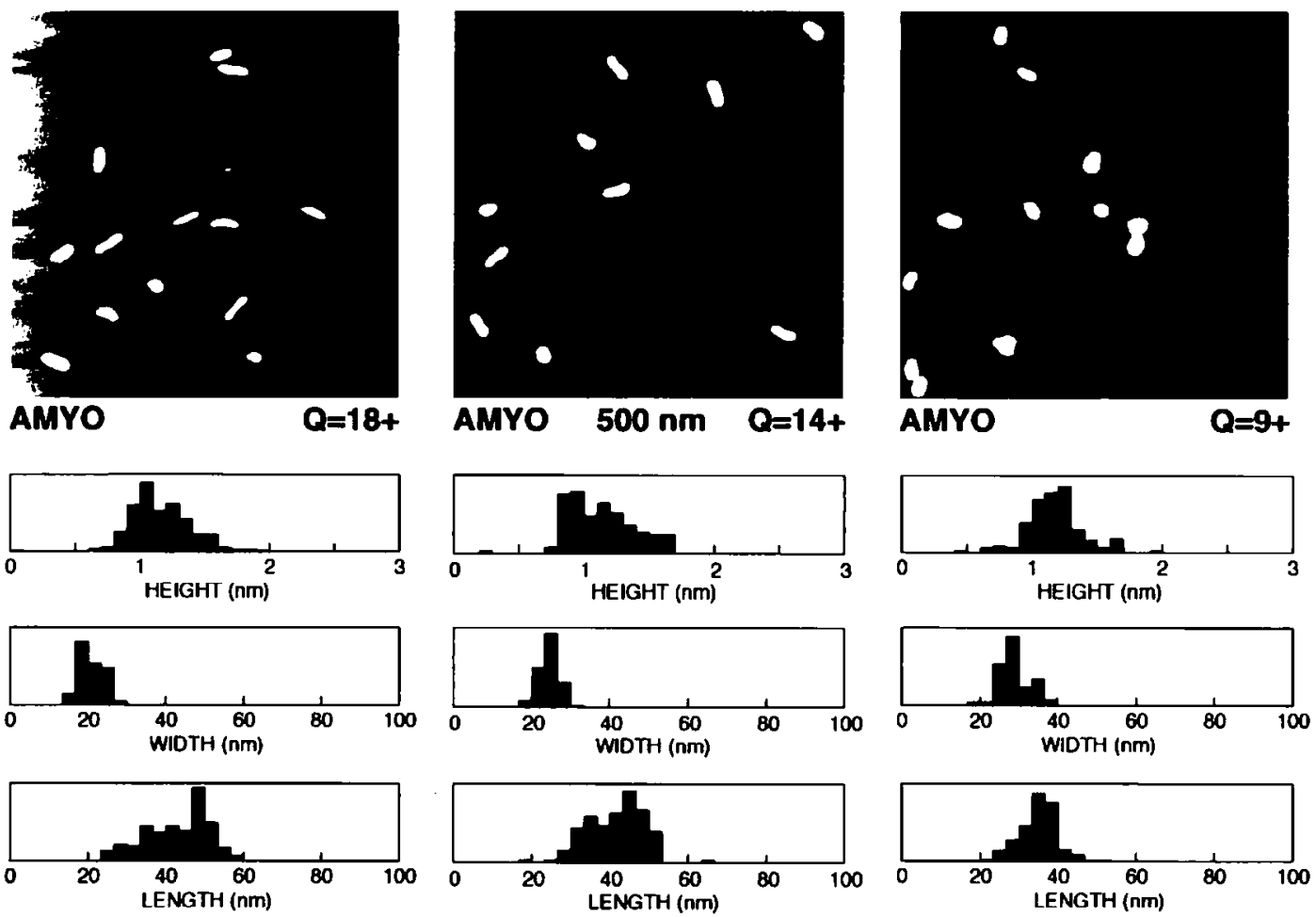

Figure 4. Typical TM-SFM images of hillocks created by impacts of $176-\mathrm{keV}$ apomyoglobin ions in various charge states onto HOPG. Histograms of hillock dimensions appear below each image. Left: $Q=+18$; middle: $Q=+14$; right: $Q=+9$. Notice that projectiles with higher charge state generate more extended hillocks. For $\mathrm{AMYO}^{9+}$, some partial delamination of the layered HOPG structure seems to have occurred about the middle hillocks in the image shown. $(Q=+18: 205$ hillocks analyzed; $Q=+14$ : 111 hillocks analyzed; $Q=+9$ : 123 hillocks analyzed.) 

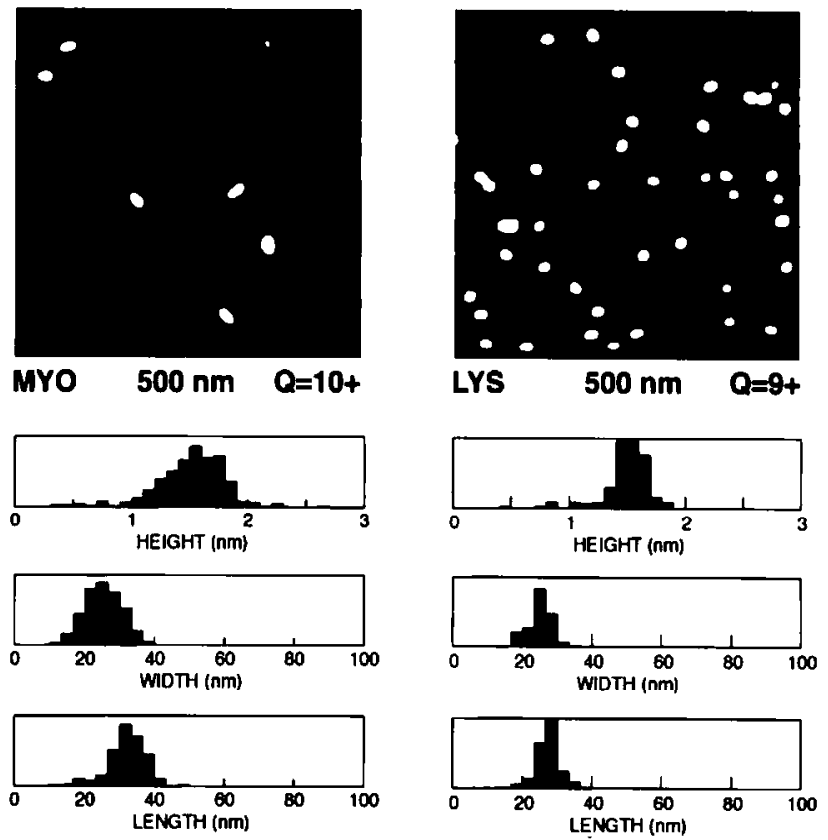

Figure 5. Typical TM-SFM images of hillocks created by impacts of 198-keV myoglobin ions with charge state $Q=+10$ (left) and 146-keV lysozyme ions with charge state $Q=+9$ (right). Corresponding histograms of hillock dimensions are shown below each image. (MYO ${ }^{10+}: 311$ hillocks analyzed; YYS $^{4+}$ : 111 hillocks analyzed.)

of the hillocks from AMYO ${ }^{18+.16+, 14+}$ bombarded samples were skewed: elongated hillocks were more common than circular hillocks, and lower hillocks were more common than taller hillocks. For AMYO projectiles, estimates for the most probable (skewed distributions) or median (symmetric distributions) values of hillock height, width, and length are plotted (Figure 6a). The $\mathrm{AMYO}^{16+}$ point was repeated for two samples and three different SFM probe tips, which gave very different values for length and width. However, the quantity length minus width was roughly constant (Figure 6b).

\section{Discussion}

\section{Scanning Probe Microscopy Artefacts}

Recent reviews summarize the operating principles of STM [33] and the various modes of SFM [31]. STM images represent a convolution of surface topography and surface energy density of states. Since we do not know whether the electronic structure of hillock material differs from that of the surrounding HOPG, we cannot a priori ascribe the observed STM images (Figure 2) to pure topography. STM studies on HOPG have produced a variety of imaging artefacts that effectively mimic nanometer-scale entities such as DNA and biomolecules [34]. Likewise, CM-SFM is sensitive to some combination of lateral forces (friction) and elastic deformation along with topography. Even TMSFM, which supposedly offers much lower contact forces and more reliable topographical imaging [30],


Figure 6. (a) Hillock length ( $L$ ), width ( $i$ ), and height $(h)$ plotted versus charge state for apomyoglobin ions incident on HOPG. The plotted values are deduced as described in the text from histograms (Figure 4) and a similar histogram for $Q=+16$. The error is taken as plus/minus one bin width (Figure 4). The bars show how the difference $L-w$ varies with charge state. (b) For $Q=+16$, hillock length and width are shown for different samples and different probe tips. (i) One sample-probe-tip combination; (ii) and (iii) another sample measured with two different probe tips. The quantity $L-w$ is roughly constant although $L$ and $w$ vary drastically. (c) Schematic diagram of the apparent broadening of hillock width $w$ and length $L$ by a contribution from the finite size of the probe tip of radius of curvature $R$. If the hillock ends and middles have a similar radius of curvature, the length and width broadening are essentially equal, which explains why $L-w$ is constant for different probe tips.

may also be probing local nanometer-scale modulations in inelastic tip-surface deformations [35] and not just topography. For adsorbed proteins, TM-SFM greatly underestimates the expected protein heights [35].

To evaluate the degree of confidence we could ascribe to our interpretations of scanning probe microscopy images, we compare results obtained with different microscopy modes and different sample types. For example, Figure 2 shows that on HOPG, we observed hillocks in two microscope modes (TM-SFM 
and STM) that function based on completely different physical principles. This result strongly implies that the hillocks are in fact topological. However, the hillock height distributions differed somewhat between STM and TM-SFM. Furthermore, the histograms show that the hillocks observed with TM-SFM were consistently wider and longer by about $10 \mathrm{~nm}$ than those measured with STM (Figure 2). This can reflect a difference in physical size between the TM-SFM and STM probe tips employed in acquisition of those data sets, as discussed in subsequent text.

A further check for artefacts is to bombard and then probe different materials, such as mica and HOPG (Figure 3). Hillocks observed on the mica samples with TM-SFM displayed similar statistical length and width distributions as compared to hillocks observed on the HOPG samples, which strongly implies that our qualitative observations on HOPG are not dominated by SFM or STM imaging artefacts. The observation of smooth hillocks in HOPG but hillocks decorated by apparent craters in mica is interesting and could reflect a variety of possibilities. The material response may differ between mica and HOPG. Also, the hardness profile off- to on-hillock may be quite different for mica and HOPG, which vary greatly in hardness. These two systems are in need of further investigation.

In scanning probe microscopy, the physical size of a probe tip is convoluted with the topology of the surface to produce images of localized features that are broadened with respect to the size and shape of the actual feature on the surface. In SFM, tip-induced broadening is often explained in terms of a simple steric convolution between the sample and the probe tip, both assumed to be incompressible [36]. Let a tip of radius of curvature $R$ probe a hillock of width $w$ and height $h$. Then the hillock width is broadened by $\Delta w \approx 2 R h w^{-1}$ (Figure 6c). Let us assume that the hillock widths measured by STM are more typical of actual hillock dimensions, because tunneling in STM is exponential in the surface-tip gap separation and because tunneling therefore often occurs from a small cluster of atoms on the tip apex. From Figure 2, hillock widths probed by TM-SFM were $\Delta w \approx 10 \mathrm{~nm}$ wider than the hillock widths probed by STM. Thus, given $h \approx 1.5 \mathrm{~nm}$, the tip radius of curvature in TM-SFM is $R \approx 60 \mathrm{~nm}$, six times the nominal value specified for probe tips of that type! In an independent study, only one in three such tips was found to be characterized by $R \approx 10 \mathrm{~nm}$ [37].

Variations in probe-tip profiles are obviously a liability in the application of SFM techniques to the study of nanometer-scale surface defects. This is demonstrated by the results of our analyses of two samples bombarded by $176-\mathrm{keV} \mathrm{AMYO}^{16+}$ and probed with three different TM-SFM probe tips. Characteristic apparent values of hillock length and width, deduced from histograms, varied considerably from case to case (Figure 6b). However, the difference between length and width appeared to be about the same in all cases
(Figure 6b). If the hillock ends have a radius of curvature similar to that of the hillock middles, which is roughly true, then image broadening due to the probetip size increases the apparent lengths by about as much as it increases the apparent widths (Figure 6c), which accounts for the constant difference (Figure 6b). Thus, the difference between observed hillock length and width is, useful in consideration of trends in data acquired from different samples and with different SFM probe tips.

More work is required to understand SFM and STM imaging contrast mechanisms. Below, we regard hillock dimensions obtained by TM-SFM to be qualitative in nature, whereas we assume that STM images provide more accurate, semiquantitative measures of hillock dimensions. Our assumption is based on the fact that hillocks in HOPG appear to be significantly narrower when imaged by STM, which suggests a higher imaging resolution.

\section{Solution-Phase Conformations of LYS, MYO, and $A M Y O$}

For this study, we employed the proteins LYS, MYO, and AMYO. The structures and conformations of LYS and MYO proteins have been determined by using $X$-ray crystallography. LYS, for example, possesses four covalent disulfide bonds that cross-link and act to stabilize the protein polypeptide chain. Consequently, LYS is very compact $(3 \times 3 \times 4.5 \mathrm{~nm})$ and stable in the native state [38]. ESI mass spectra of LYS display low charge state distributions [10], consistent with LYS being compact, because the charge on a protein is believed to scale with the protein's degree of extension [6]. Furthermore, only small changes occur in the ESI mass spectrometry distribution of charge states when solution $\mathrm{pH}[8]$ and temperature $[39,40]$ are changed; these facts are taken as further evidence for the stability of LYS.

Although the globular protein MYO is slightly more massive than LYS, it is also very compact in its native state $(2.5 \times 3.5 \times 4.5 \mathrm{~nm})$ [38]. MYO possesses no disulfide bonds, but under physiological conditions it is stabilized by both a noncovalently bound heme group and by nonpolar interactions between water molecules and polar-nonpolar amino acids arrayed in a special configuration [38]. The structures for MYO in crystalline form and under physiological conditions in solution are very similar [38]. By lowering solution $\mathrm{pH}$, the heme unit can become detached from MYO to form AMYO, a somewhat less massive molecule. The helical content is reduced for AMYO, and hydrodynamic studies suggest that AMYO is less compact than MYO (page 57 in ref 38). Charge-state distributions of electrosprayed MYO and AMYO ions tend to bear out these structural features: MYO is often produced in significantly lower charge states than AMYO (Figure 1), consistent with a greater compactness of MYO in solution. Under some conditions, AMYO displays a 
bimodal charge-state distribution [20,41], each contribution to which is thought to represent a different conformation of the AMYO protein. When sequentially raising the $\mathrm{pH}$ of an AMYO solution, the ESI chargestate distribution first shifts to lower charge as the protein refolds and then shifts to a higher mass as the heme group is reattached noncovalently to the protein [41].

\section{Conformation of $A M Y O^{18+, 16+, 14+}$}

Collision cross sections for proteins have been derived based on their energy loss when they pass through a collision cell that contains argon gas [18, 19]. The collision cross section for AMYO increased smoothly and monotonically with the charge state of the protein [18]. In another collision cell study [20], the collision cross section for AMYO ions characterized by a charge-state distribution centered around $Q=+20$ was found to be larger than the collision cross section for AMYO ions characterized by a lower charge-state distribution centered around $Q=+10$. These results suggest that AMYO and other proteins typically are physically larger when they are more highly charged. However, different protein shapes would not necessarily yield different collision cross sections, because protein orientation can be a significant factor in determination of collision cross sections for nonglobular proteins $[18,19]$.

Highly charged protein ions have been trapped in a Fourier-transform ion cyclotron resonance (FTICR) mass spectrometer and both the number of accessible labile $\mathrm{H}$ atoms and the rate constants for hydrogen-deuterium exchange in the gas phase have been monitored [42]. AMYO in charge states that range from $Q=+12$ to +16 displayed roughly the same number of reactive hydrogens, about 72 , which corresponds to about $26 \%$ of the total number of theoretically exchangeable hydrogens. The exchange rate constant was found to be insensitive to the charge state of the protein [42]. To the extent that the number of exchanged hydrogens is an indication of the openness of the protein structure, the FTICR observations [42] seem to imply that the structure of AMYO is independent of charge state, in contradiction to the results of gas-phase scattering experiments $[18,19]$.

For a polypeptide chain with 153 amino acids and $60 \% \alpha$-helicity (liquid-phase AMYO, ref 38), about 90 theoretically exchangeable hydrogens that participate in the helices probably would not undergo rapid hydrogen-deuterium exchange, which leaves 63 exchangeable hydrogens along the polypeptide backbone. Oxygen-bound hydrogens on the amino acid side chains may be exchanged much more efficiently than nitrogen-bound hydrogens [42], which gives an additional 15 exchangeable hydrogens for a total of 78; this is not far from the observed exchange level of 72 in the gas phase [42]. This simple estimation seems to imply that much of the $\alpha$-helicity of highly charged
AMYO is preserved in the gas-phase environment. Thus, the discrepancy between the two experimental results may be resolved by supposing that (1) hemeless AMYO is not constrained to the normal compact configuration characteristic of $\mathrm{MYO}$, and therefore AMYO expands somewhat to give higher collision cross sections and (2) the expansion occurs without loss of $\alpha$-helicity, to give relatively charge-state-independent hydrogen-deuterium exchange characteristics.

We have observed rather extended, oblong hillocks generated by $\mathrm{AMYO}^{18+, 16+, 14+}$ projectiles (Figure 4). If we assume that the hillock shape is correlated with the conformation of the gas-phase protein ion, our results indicate that AMYO in high charge states possesses a relatively extended conformation. The length distributions of these hillocks were skewed toward higher length values (Figure 4). If we assume that "sticklike" molecules are randomly oriented in space, solid-angle considerations dictate that "side-on" impacts are more common then "end-on" impacts (Appendix A). Therefore, the skewed length distributions of the hillocks generated by the higher charge states of AMYO $\mathrm{O}^{18+, 16+, 14+}$ are consistent with these projectiles being extended, tubular, or sticklike. Moreover, the hillocks generated by the lower-charge state $\mathrm{AMYO}^{9+}$ projectiles are more compact than those generated by the higher charge states AMYO ${ }^{18+, 16+, 14+}$ (Figure 4).

Hillock length and width are plotted versus AMYO charge state (Figure 6a). In the foregoing text we discussed the drawback that the observed lengths and widths are strongly perturbed by the size of the SFM probe tip, but we noted that general trends in data can be observed safely by noting the behavior of the quantity length minus width, which was also shown (Figure 6a). Length minus width appears to monotonically increase with the AMYO charge state, in qualitative agreement with gas-phase scattering results $[18,19]$. We thus suggest that the gas-phase scattering results are more indicative of gross molecular structure, whereas the hydrogen-deuterium exchange results probe more subtle aspects of protein conformation, which include the effect of the structure and charge state on exchange rates [42].

\section{Conformations of $\mathrm{AMYO}^{9+}$ and $M Y \mathrm{O}^{10+}$}

Gas-phase reactions that involve electrosprayed proteins from solutions in which these proteins were either in the native form or were denatured have been monitored [14, 15]. Prior to mass-to-charge ratio analysis in a mass spectrometer, the electrosprayed ions were allowed to interact with either trimethylamine, triethylamine, or 1,6-hexanediamine. Because these last three molecules bind protons more strongly than adducted protons are bound to the electrosprayed protein ions, the interaction results in the partial deprotonation of the protein molecular ions. No significant difference in reactivity existed between the lower 
charge states of AMYO $(Q=+4)$ and $\mathrm{MYO}(Q=+5)$ [14]. These results $[14,15]$ are compatible with the hypothesis that both MYO and AMYO in low charge states possess similar gas-phase structures.

Our results show visually that the shapes and sizes of AMYO and MYO in the lower charge states are similar (Figures 4 and 5). If the difference between hillock lengths and widths implied by the respective histograms is considered, there is no basis to argue from our data that there is any difference between the conformations of AMYO and MYO for our ESI source conditions. Both the $\mathrm{AMYO}^{9+}$ and the $\mathrm{MYO}^{10+}$ projectiles generated rather compact, nearly circular hillocks; these features were significantly more compact than those created by AMYO ${ }^{18+, 16+, 14+}$.

\section{Conformation of $L Y S^{10+}$}

Egg-white lysozyme and bovine albumin, electrosprayed from solutions in which these proteins were either in the native form or were denatured by reduction of the structure-stabilizing disulfide bonds, have been allowed to undergo deprotonation reactions with diethylamine prior to mass-to-charge ratio analysis in a mass spectrometer [43]. The key result of this study was that highly charged gas-phase species that originate from native solution-phase conformers react more strongly with diethylamine to form lower final charge states than do gas-phase species that originate from denatured solution-phase conformers. No rigorous conclusions were reached about the structure of gas-phase electrosprayed ions [43]. However, it was suggested that Coulomb repulsion and hence proton transfer exothermity from a tightly folded conformer would exceed that of a more extended conformer. Consequently, the experimental results could be rationalized by assuming that the electrosprayed ions retain significant features of the higher order structure they possessed in solution.

Our results visually support part of this rationalization. We electrosprayed LYS from a solution in which the proteins were in the native form. These projectiles generated compact features on HOPG, which indicates that the gas-phase highly charged $\mathrm{LYS}^{9+}$ ions possessed a compact conformation. Of the three projectiles that produced compact hillocks-AMYO ${ }^{9+}, \mathrm{MYO}^{10+}$ and $\mathrm{LYS}^{9+}-\mathrm{LYS}^{9+}$ produced the most compact hillocks, completely in keeping with its highly stabilized disulfide-interlinked polypeptide chain.

In a previous work of ours, tentative results were reported on similar impact experiments performed with native and disulfide-reduced albumin [26]. Impact hillocks formed with electrosprayed native albumin were somewhat elongated, consistent with partial Coulombic-driven denaturation of this three-domain blood protein [44]. With disulfide bond reduction, each of the three domains of the albumin protein evidently was destabilized significantly, because the resulting impact hillocks appeared to be much longer than what was observed for native albumin. These results also are consistent with the interpretation of the gas-phase deprotonation results [43].

In another previous work, secondary ion emission from targets bombarded by energetic protein ions was discussed [28]. The multiplicity, that is, the total number of different types of emitted secondary ions under primary protein ion impact, was found to be anomalously high for AMYO when compared to LYS or to albumin. If we assume that secondary ions originated only from the near-surface layers of the impacted target, the results were rationalized by supposition that the spread-out AMYO simply does not penetrate as far into the sample as does the compact LYS. Consequently, for the same delivered kinetic energy, a larger fraction of the secondary ions induced by AMYO escape than do for LYS. These results are in qualitative agreement with the results of the present study.

\section{The Length of Gas-Phase AMYO ${ }^{14+}$}

The extraction of specific dimensions of the gas-phase proteins requires certain assumptions about what TMSFM and STM are really measuring. There is considerable uncertainty in this regard. We have assumed that STM images provide inherently more accurate lateral dimensions of hillocks on HOPG, simply because these hillocks appeared narrowest when working with STM. We also must consider the response of the surface to an impacting energetic polyatomic projectile. In the impact speed regime employed in the present work, the projectile interacts collisionally with the target and deposits its energy within 1-3 nm of the surface, as shown in recent experiments with oxidative defect titration on HOPG [45]. Impacts of polyatomic projectiles with surfaces generate unique transient conditions of high temperature and pressure [46]. Such impacts have been modeled in terms of an evaporative thermal spike model for amorphous thin carbon foils [47].

In Appendix B, we outline a thermal model in which deposition of energy in a line along the surface results in the formation of a melt zone that, when resolidification rapidly occurs, leaves a hillock composed of amorphous carbon. Because of energy diffusion, the hillock is wider and longer than the geometrical projection of the incident molecular ion onto the surface. When fitting a suitable formulation of this model to the results obtained for 252-keV AMYO ${ }^{14+}$ (Figure 2), we find that we can obtain a reasonable fit with $L=35.5 \pm 2.4 \mathrm{~nm}$. For an $\alpha$-helix, the length per amino acid residue is $0.15 \mathrm{~nm}$ residue $^{-1}$, and for completely stretched-out polypeptide chain, this figure is $0.36 \mathrm{~nm}$ residue $^{-1}$. AMYO possesses 153 amino acid residuals and in solution is characterized by a helical content of $\approx 60 \%$ [38]. AMYO stretched out as much as possible without destruction of the $\alpha$-helices thus would be characterized by a length of around $36 \mathrm{~nm}$, 
close to the length we observe. A $60 \%$ gas-phase helical content does not seem inconsistent with the observation of 72 exchanged hydrogens in FTICR experiments described in the foregoing text [42]. The width of an $\alpha$-helix is on the order of $1 \mathrm{~nm}$, so using the value of $L$ we obtained the gas-phase collision cross section is predicted to be around $35.5 \mathrm{~nm}^{2}$, which is in good qualitative agreement with observed gas-phase collision cross sections $[18,19]$.

In the gas-phase collision experiments [18, 19], random orientation and collisionally induced random changes in orientation of sticklike AMYO could be assumed. Then the same solid-angle considerations of the falling-stick model (Appendix A) also would imply that the gas-phase collisions mainly involve AMYO oriented with the long axis generally perpendicular to the direction of propagation through the gas cell. Thus, impact features presented here and gas-phase scattering data $[18,19]$ qualitatively measure the same molecular geometry.

\section{Conclusions and Prospects}

Qualitative structural information about gas-phase protein ions produced by electrospray ionization has been derived from tapping-mode scanning force and scanning tunneling microscopy images of impact defects generated by individual energetic proteins incident on highly oriented pyrolytic graphite (HOPG). This new approach to the study of the conformation of gas-phase protein ions employs an unique visually oriented technique, which complements the approaches represented by other gas-phase studies.

The impact defect imaging technique employed in the present work has some limitations. For compact ions, the method only identifies the projectiles as being relatively compact, and the trends one observes are essentially qualitative in nature. It is difficult to derive quantitative information on compact protein ions, although with suitable modeling, it is possible to extract numerical information for more extended protein ions. Nonetheless, we believe that three improvements may provide more and much higher resolution data. One approach is to considerably reduce the impact kinetic energy. This means that the disturbed region around the impact point, which is imaged by microscopy, will be more comparable in size to the incident ion itself. Another approach is to focus exclusively on highresolution imaging by scanning tunneling microscopy, using high quality sharpened and characterized probe tips, generated and deployed under vacuum conditions. Last, pattern recognition and numerical image analysis software may significantly speed up the process of microscope images analysis. With such improvements, the impact defect imaging technique could be applied to evidently more complex problems, such as examination of the multiple coexisting conformers of cytochrome $c[17,42]$.

\section{Appendix A: The Falling-Stick Model}

Consider an elongated, sticklike molecule of length $L$ that approaches a surface in an orientation characterized by polar angle $\theta$ and azimuthal angle $\varphi$ (Figure 7). We assume that this impacting molecule will leave an imprint in the surface of length $l=L \sin (\theta)$. We desire to know the distribution of the length $l$ for a large ensemble of impacting molecules of random orientation.

Let $f(\theta, \phi)$ be the fraction of the molecules pointing within a unit solid angle. Random orientation and normalization imply that this fraction is simply constant, $f(\theta, \phi)=1 / 2 \pi$ (note that the molecule is assumed symmetric). So, the fraction of all molecules pointing within a differential solid angle $d \Omega$ is $f(\theta, \phi) d \Omega=d \Omega / 2 \pi$. The differential solid angle is $d \Omega=-d \phi d[\cos (\theta)]$. The form of this differential and the fact that $f(\theta, \phi)$ is constant imply that for the falling stick, the variable $\phi$ is uniformly randomly distributed in the interval $[0,2 \pi]$, while the variable $[\cos (\theta)]$ is uniformly randomly distributed in the interval $[-1,1]$. We wish to find the distribution $g(l)$ in $l=L \sin (\theta) . g(l) d l$ is the fraction of all impacts that give a projected length on the surface in the range $[l, l+d l]$.

A very simple way to derive a representation of $g(l)$ is to do so numerically. With a spreadsheet program, we filled a large array with random numbers between -1 and 1 . These represented the randomly distributed values of $[\cos (\theta)]$. We then converted each array entry to $l=L \sin (\theta)$, which then represented the projected lengths of the impacting molecules onto the surface. (Note that the projected length $l$ does not depend on $\phi$.) Finally, the $l$ values were histogramed into bins of width $\Delta l$ and the histogram $N(l)$ was plotted (Figure 8 ). $N(l)$ is the number of projected

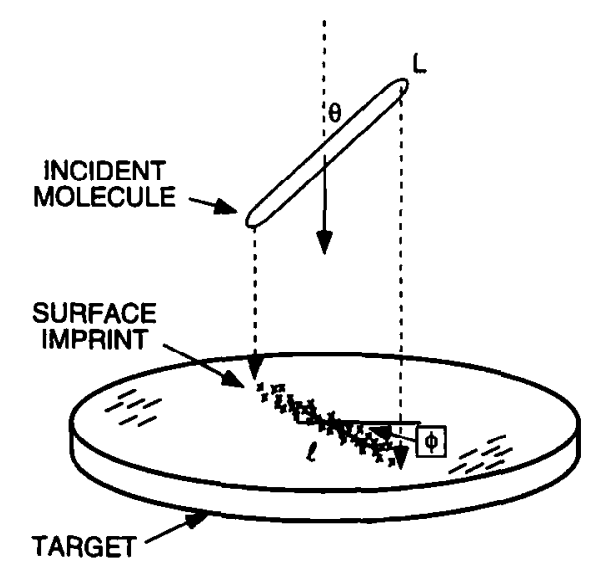

Figure 7. Impact geometry and coordinate system for the "falling-stick" model of the impact of an elongated protein projectile onto a surface. $\theta$ is the polar angle and $\phi$ is the azimuthal angle in spherical coordinates. $L$ is the length of the elongated projectile and $l$ is the projected length of the projectile onto the surface. $l=L \sin (\theta)$. 

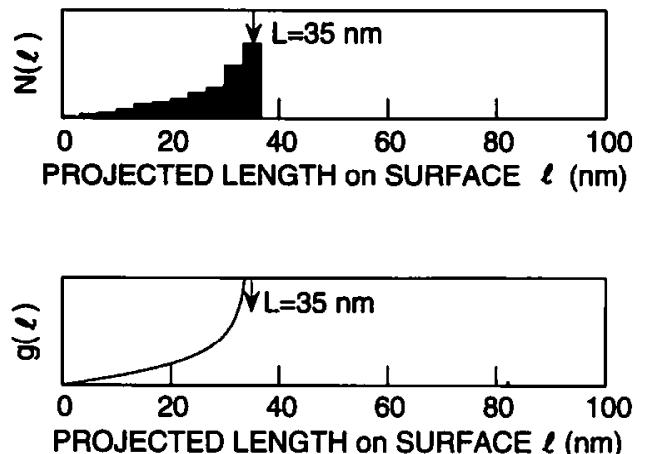

Figure 8. Distributions of the projected lengths of falling sticks of length $35 \mathrm{~nm}$. Top: Numerical solution in occupancy per histogram bin with bin width $3.33 \mathrm{~nm}$. Bottom: Normalized analytical solution expressed in fraction occupancy per unit projected length interval (i.e., no histograming). The two plots reflect the statistics of random orientation of a stick above a surface: There are more ways to achieve parallel orientation than to achieve perpendicular orientation.

lengths lying in the interval $[l, l+\Delta l]$. The resulting distribution is skewed to higher values of projected length $l$.

Analytically, it may be shown that

$$
g(l)=\frac{l}{L} \frac{1}{\sqrt{L^{2}-l^{2}}}
$$

$g(l)$ (Figure 8 ) diverges when $l=L$, but by integrating between $l=0$ and $l=L$ it can be seen that the distribution is properly normalized to an area of 1 . The distribution clearly is skewed to larger values of length l. The numerical and analytic approaches are equivalent in the limit of a very large number $N_{0}$ of impact events. For sufficiently small bin sizes $\Delta l, N_{0}^{-1} N(l)$ approaches $g(l) \Delta l$.

A complication in the falling-stick model is that sticklike molecular ions are likely to be tumbling through space and do not approach the target surface in fixed orientation $(\theta, \phi)$. However, during the subpicosecond time scale over which the molecule-surface collision typically takes place, these angles change only minutely. Furthermore, a convolution of tumbling motion with a randomly oriented axis of rotation leads to a randomly oriented long molecular axis (Figure 7) and, hence, to the same projected length distribution as calculated previously.

If the molecular ions are not charged uniformly along their lengths, then the acceleration electric field induces a more complex motion due to a time- and orientation-dependent torque. We have not treated the resulting motion in full detail. However, in a simplified version of this problem, an oscillatory (indeed approximately sinusoidal) motion in variable $\theta$ (Figure 7) results. This motion enhances the probability of observing end-on impacts characterized by $\theta \approx 0^{\circ}$, but side-on impacts characterized by $\theta \approx 90^{\circ}$ are still fa- vored by about a factor of 2 . The distribution of projected lengths is still skewed to higher values.

As a highly charged molecular ion approaches a conducting surface, it also starts to interact attractively with its image charge [22] and, again, if the molecular charge is distributed nonuniformly along the length of the molecule, a transient torque acts on the molecular ion. However, this torque is highest just before the molecule impacts the surface moving at its highest speed. We estimated that the molecule does not have a sufficiently large amount of time to react significantly to the torque.

The skewed distributions shown in Figure 8 are seen to be a consequence of the statistics of random orientation. For an extended, sticklike molecule, there are many more orientations generally parallel to the surface than there are orientations generally perpendicular to the surface. Our observations of hillocks significantly skewed to longer lengths for $\mathrm{AMYO}^{18+, 16+, 14+}$ (Figure 4) indeed imply that these molecules are extended in a sticklike fashion. The length distributions observed in the present work (Figure 4) are skewed but are also rounded, not completely resembling the falling-stick distributions (Figure 8). This is not surprising: as discussed in Appendix $B$, the surface is modified during diffusion of the energy deposited by an impacting molecular ion, which leads to a longer and wider area of hillock formation than just the area defined by the projection of the ion onto the surface. Also, there may be a distribution of different conformations present in an ensemble of molecular ions. Higher-resolution surface imaging combined with suitable modeling may allow this distribution of conformer shapes to be resolved.

\section{Appendix B: Thermal Spike Model for Hillock Formation}

Recently, a simple thermal spike model of latent track formation in ordered magnetic insulators bombarded by megaelectronvolt atomic ions has been presented [48]. The model simply states that a certain region around an ion track is melted and is subsequently quenched so rapidly that it solidifies in an amorphous state, which has a different density and therefore generates topological relief on the surface. In this model, the amorphous region is defined by the maximum extent of the region of the material that melted at any time under the evolution of the thermal spike. HOPG is a crystalline material that melts at temperature $T_{m} \approx$ $4200 \mathrm{~K}$ [49]. The hillocks observed in the present work can represent regions of the HOPG that melted and resolidified to an amorphous state in a nanometer-scale region surrounding each energetic protein impact. Let us apply the model of ref [48] to the HOPG hillocks.

Graphite is an unusual material that features extremely anisotropic thermal conductivity: $30 \mathrm{~W} \mathrm{~cm}^{-1}$ $\mathrm{K}^{-1}$ in the basal plane versus $0.06 \mathrm{~W} \mathrm{~cm}-1 \mathrm{~K}^{-1}$ normal to the basal plane [49]. We thus employ a two- 
dimensional in-basal-plane dissipationless thermal diffusion model for the energy density $\varepsilon(r, t)$ at the surface around a point at which energy has been deposited (Figure 9):

$$
\frac{1}{r} \frac{\partial}{\partial r}\left(r \frac{\partial}{\partial r}\right) \varepsilon(r, t)=\frac{1}{\kappa} \frac{\partial}{\partial t} \varepsilon(r, t)
$$

$\kappa$ is the thermal diffusivity in the basal plane of HOPG. The thermal spike diffusion equation (eq 1) is frequently cast in terms of temperature, but the meaning of temperature is unclear in the present context. In the impact region, thermal equilibrium does not necessarily exist. Furthermore, most of the energy needed to melt graphite is taken up to induce the phase transition (2.1 eV per atom) rather than to heat up the graphite to its melting temperature $(0.34 \mathrm{eV}$ per atom). A full treatment of this complex problem is beyond the scope of the present work. Thus, we express the diffusion equation quite generally in terms of energy density and furthermore assume that the thermal diffusivity is independent of this energy density. The model, though simplified, should suffice as a means to first approach the problem at hand.

Let energy $\Delta E_{i}$ be deposited in the first layer of the HOPG, which is of lattice spacing $l_{c}$ perpendicular to the basal plane. Equation 1 is solved by the following expression for $\varepsilon(r, t)$ :

$$
\varepsilon(r, t)=\frac{\Delta E_{i}}{l_{c} 4 \pi \kappa t} \exp \left(-\frac{r^{2}}{4 \kappa t}\right)
$$

Equation 2 holds for an infinitesimal initially energized region of the target. For a line source more appropriate for extended projectiles (Figure 7), eq 2 must be modified. We assume that the composite energy density is a summation of an appropriate collection of miniature point sources of heat located along the impact line. Thus, in the spirit of [50], we integrate eq 2 over the geometry shown in Figure 9, to obtain:

$$
\begin{aligned}
\varepsilon(\rho, \zeta, t)= & \frac{1}{\sqrt{2 \pi}} \frac{\Delta E_{i}}{L l_{c}} \frac{1}{\sqrt{4 \kappa t}} \exp \left(-\frac{\rho^{2}}{4 \kappa t}\right) \\
& \times\left[\operatorname{erf}\left(\frac{L-2 \zeta}{2 \sqrt{4 \kappa t}}\right)+\operatorname{erf}\left(\frac{L+2 \zeta}{2 \sqrt{4 \kappa t}}\right)\right]
\end{aligned}
$$

With this equation, the maximum region of melt has to be calculated numerically. In this simple model, the value of $\kappa$ does not matter, because it affects only when the maximum temperature is reached, not what that maximum temperature is. An energy density for melting consistent with $2.4 \mathrm{eV}$ per atom is utilized in the calculation.

As shown in Figure 2, $\mathrm{AMYO}^{14+}$ ions that impacted with kinetic energy $252 \mathrm{keV}$ generated elongated hillocks. We replot the data with the highest available resolution, the STM data, in such a fashion as to show clearly which dimensions the longest hillocks had (Figure 10). We assume that this high-resolution data contains a negligible contribution from the probe-tip size. The longest hillocks were also the most narrow (Figure 10). A cluster of points was selected with lengths located roughly in the middle of the high length falloff of the length histogram. This cluster of defects was characterized by lengths in the range $38-41 \mathrm{~nm}$ and widths in the range $13-17 \mathrm{~nm}$. We regarded from the histograms that these ranges are quite representative of the longest defects on the surface, when we take into account that there is likely to be some spread in the defect dimensions for otherwise identical impacts. There is a specific range of the parameters $\Delta E_{i}$ and $L$ with which the simple model can simultaneously describe these hillock lengths and widths for these hillocks, and the resulting value of $L$ is $35.5 \pm 2.4 \mathrm{~nm}$. This value is discussed in the main text. The very longest hillock observed was $42.3 \mathrm{~nm}$ long and $13.7 \mathrm{~nm}$ wide. For this hillock, $L=38.5 \mathrm{~nm}$. In future work, we will attempt to completely deconvolute defect length and width histograms into contributions from molecular ion orientation and conformation, and probe-tip radius.
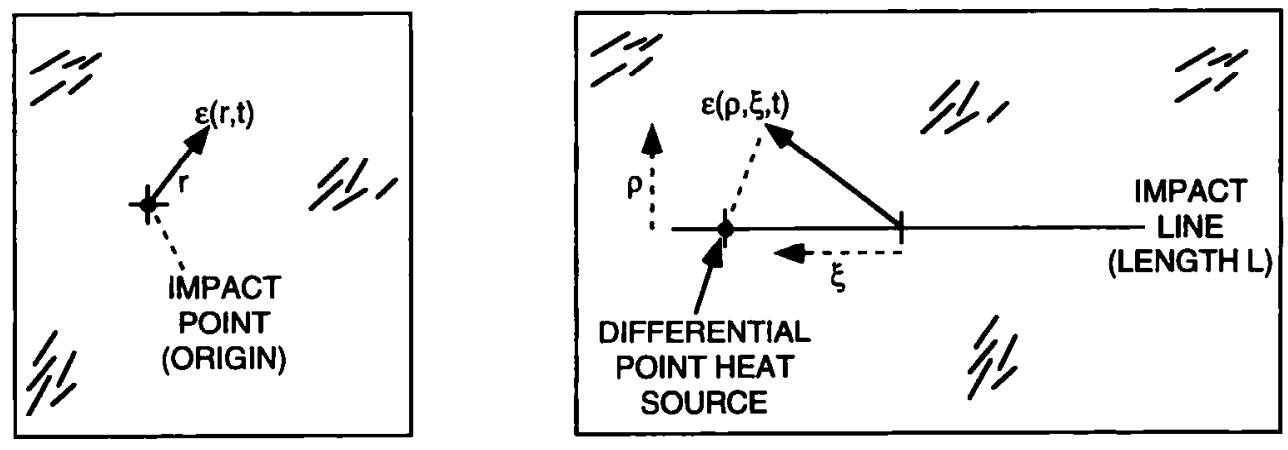

TARGETS (TOP VIEW)

Figure 9. Geometry of the thermal spike model. Left: Geometry for energy initially deposited in an infinitesimal spot on the surface. Right: Geometry for energy initially deposited in an infinitesimally narrow line of length $L$. 


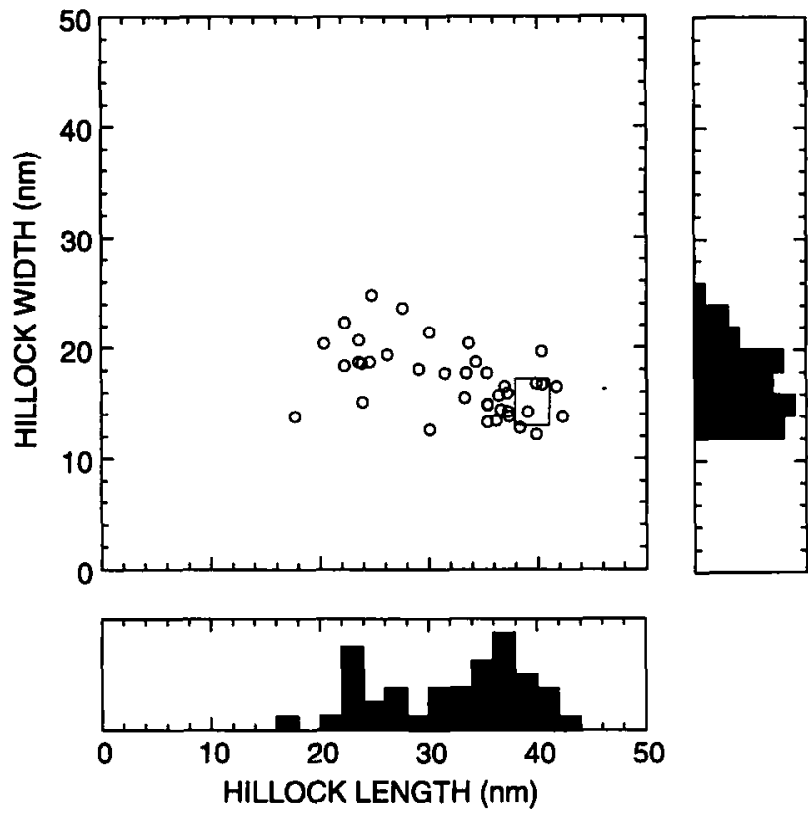

Figure 10. Hillock width plotted versus hillock length for $\mathrm{AMYO}^{14+}$ ions incident on HOPG at $252 \mathrm{keV}$ and measured with STM. Length and width histograms also are shown. The box indicates the region of width-length space that encompasses the long hillocks on the upper edge of the length histogram. This area is used to achieve a fit of the thermal spike model to obtain a value for the length of the impacting $A M Y O^{1+}$, as discussed in the text.

\section{Acknowledgments}

The authors thank the Swedish Research Council for Engineering Sciences (TFR), the Knut and Alice Wallenberg Foundation, the Swedish National Board for Industrial and Technical Development (NUTEK), and the Göran Gustafsson Foundation. One of the authors (SA) was an ERASMUS exchange student from the University of Heidelberg. Petter Lindberg's inciteful derivation of the solution to the "falling-stick" problem also is gratefully acknowledged, along with the technical assistance of J. Kjellberg, S.-O. Linder, and M. Sparby. We also acknowledge helpful discussions with R. Zubarev, I. Bitensky, P. Roepstorff, P. Demirev, and $P$. Håkansson.

\section{References}

1. Fenn, J. B.; Mann, M.; Meng, C. K.; Wong, S. F.; Whitehouse, C. M. Science 1989, 246, 64-71.

2. Meng, C. K.; Mann, M.; Fenn, J. B. Z. Phys. D 1988, 10 , 361-368.

3. Mann, M. Org. Mass Spectrom. 1990, 25, 575-587.

4. Smith, R. D.; Loo, J. A.; Edmonds, C. G.; Barinaga, C. J.; Udseth, H. R. Anal. Chem. 1990, 62, 882-899.

5. Chen, R.; Cheng, X.; Mitchell, D. W.; Hofstadler, S. A.; Wu, Q.; Rockwood, A. L.; Sherman, M.; Smith, R. D. Anal. Chem. 1995, 67, 1159-1163.

6. Loo, J. A.; Edmonds, C. G.; Udseth, H. R.; Smith, R. D. Anal. Chem. 1990, 62, 693-698.

7. Chowdhury, S. K.; Katta, V.; Chait, B. T. J. Am. Chem. Soc 1990, 112, 9012-9013.
8. Loo, J. A.; Loo, R. R. O.; Udseth, H. R.; Edmonds, C. G.; Smith, R. D. Rapid Commun. Mass Spectrom. 1991, 5, 101-105.

9. LeBlanc, J. C. Y.; Beuchemin, D.; Siu, K. W. M.; Guevremont, R.; Berman, S. S. Org. Mass Spectrom. 1991, 26, 831-839.

10. Hutchens, T. W.; Allen, M. H. Rapid Commun. Mass Spectrom. 1991, 6, 469-473.

11. Rockwood, A. L.; Busman, M.; Smith, R. D. Int. J. Mass Spectrom. Ion Processes 1991, 111, 103-129.

12. Loo, R. R. O.; Udseth, H. R.; Smith, R. D. J. Am. Soc. Mass Spectrom. 1992, 3, 695-705.

13. Winger, B. E.; Light-Wahl, K. J.; Smith, R. D. J. Am. Soc. Mass Spectrom. 1992, 3, 624-630.

14. Loo, R. R. O.; Winger, B. E.; Smith, R. D. J. Am. Soc. Mass Spectrom. 1994, 5, 1064-1071.

15. Loo, R. R. O.; Smith, R. D. J. Am. Soc. Mass Spectrom. 1994, 5, 207-220

16. Wagner, D. S.; Anderegg, R. J. Anal. Chem. 1994, 66, 706-711.

17. Wood, T. D.; Chorush, R. A.; Wampler, F. M., III; Little, D. P.; O'Connor, P. B.; McLafferty, F. W. Proc. Natl. Acad. Sci. USA 1995, 92, 2451-2454.

18. Covey, T. R.; Douglas, D. J. I. Am. Soc. Mass Spectrom. 1993, 4, 616-623.

19. Duuglas, D. J. J. Am. Soc. Mass Spectrom. 1994, 5, 17-18.

20. Cox, K. A.; Julian, R. K.; Cooks, R. G.; Kaiser, R. E. I. Am. Soc. Mass Spectrom. 1994, 5, 127-136.

21. Axelsson, J.; Reimann, C. T. Nucl. Instrum. Methods Phys. Res. B 1994, 93, 499-504.

22. Axelsson, J.; Parilis, E. S.; Reimann, C. T.; Sullivan, P.; Sundqvist, B. U. R. Nucl. Instrum. Methods Pliys. Res. B 1995, $101,343$.

23. Axelsson, J.; Reimann, C. T.; Sundqvist, B. U. R. Int. J. Mass Spectrom. Ion Processes 1994, 133, 141-155.

24. Axelsson, J.; Reimann, C. T.; Sundqvist, B. U. R. Nucl. Instrum. Methods Phys. Res. B 1994, 88, 131-137.

25. Quist, A. P.; Ahlbom, J.; Reimann, C. T.; Sundqvist, B. U. R. Nucl. Instrum. Methods Phys. Res. B 1994, 88, 164-169.

26. Reimann, C. T.; Quist, A. P.; Kopniczky, J.; Sundqvist, B. U. R.; Erlandsson, R.; Tengvall, P. Nucl. Instrum. Methods Phys. Res. B 1994, 88, 29-34.

27. Sullivan, P. A.; Axelsson, J.; Sundqvist, B. U. R. Rapid Commun. Mass Spectrom. 1995, 9, 377-382

28. Zubarev, R. A.; Bitensky, I. S.; Demirev, P. A.; Sundqvist, B. U. R. Nucl. Instrum. Methods Pliys. Res. B 1994, 88, 143-148.

29. Allen, M. H.; Vestal, M. L. J. Am. Soc. Mass Spectrom. 1992, 3, 18-26.

30. Zhong, Q.; Inniss, D.; Kjoller, K.; Elings, V. B. Surf. Sci. Lett. 1993, 290, L688-L692.

31. Quate, C. F. Surf. Sci. 1994, 299 /300, 980-995.

32. Bräuchle, G.; Richard-Schneider, S.; lllig, D.; Rochenberger, J.; Bech, R. D.; Kappes, M. M. Appl. Phys. Lett. 1995, 67, 52.

33. Rohrer, H. Surf. Sci. 1994, 299/300, 956-964.

34. Clemmer, C. R.; T. P. Beebe, J. Science 1991, 251, 640-642.

35. Quist, A. P.; Björck, L. P.; Reimann, C. T.; Oscarsson, S. O.; Sundqvist, B. U. R. Surf. Sci. 1995, 325, L406-L412.

36. Odin, C.; Aimé, J. P.; Kaakour, Z. E.; Bouhacina, T. Surf. Sci. 1994, 317, 321-340.

37. Sheiko, S. S.; Möller, M.; Cantow, H. J.; Magonov, S. N. Polym. Bull. 1993, 31, 693.

38. Stryer, L. Biochemistry; W. H. Freeman and Co.: San Francisco, 1981.

39. Hamdan, M.; Curcuruto, O. Rapid Commun. Mass Spectrom. 1994, 8, 144-148. 
40. Mirza, U.; Cohen, S. L.; Chait, B. T. Anal. Chem. 1993, 65, 1-6.

41. Feng, R.; Konishi, Y. I. Am. Soc. Mass Spectrom. 1993, 4, 638-645.

42. Suckau, D.; Shi, Y.; Beu, S. C.; Senko, M. W.; Quinn, J. P.; Wampler, F. M., III; McLafferty, F. W. Proc. Natl. Acad. Sci. USA 1993, 90, 790-793.

43. Loo, R. R. O.; Loo, J. A.; Udseth, H. R.; Fulton, J. L.; Smith, R. D. Rapid Commun. Mass Spectrom. 1992, 6, 159-165.

44. He, X. M.; Carter, D. C. Nature 1992, 358, 209-215.

45. Reimann, C. T.; Sullivan, P. A.; Türpitz, A.; Altmann, S.;
Quist, A. P.; Bergman, A.; Oscarsson, S. O.; Sundqvist, B. U. R.; Håkansson, P. Surf. Sci. Lett., 1995, 341, L1019-L1024. 46. Friedman, L.; Vineyard, G. H. Commun. Atom. Mol. Phys. 1984, 15, 251-259.

47. Matthew, M. W.; Beuhler, R. J.; Ledbetter, M.; Friedman, L. Nucl. Instrum. Methods Phys. Res. B 1986, 14, 448-460.

48. Szenes, G. Phys. Rev. B 1995, 51, 8026-8029.

49. Dresselhaus, M. S.; Kalish, R. Ion Implantation in Diamond, Graphite and Related Materials; Springer-Verlag: Berlin, 1992.

50. Fenyö, D.; Johnson, R. E. Phys. Rev. B 1992, 46, 5090-5099. 